\title{
Gastric lymphatic flows may change before and after endoscopic submucosal dissection: in vivo porcine survival models
}

\author{
Kyoko Nohara ${ }^{1,5} \cdot$ Osamu Goto $^{2,3} \cdot$ Hiroya Takeuchi ${ }^{1,4} \cdot$ Motoki Sasaki $^{2} \cdot$ Tadateru Maehata $^{2} \cdot$ Naohisa Yahagi $^{2}$. \\ Yuko Kitagawa $^{1}$
}

Received: 29 October 2018 / Accepted: 22 December 2018 / Published online: 2 January 2019

(c) The International Gastric Cancer Association and The Japanese Gastric Cancer Association 2019

\begin{abstract}
Background and study aim Standard gastrectomy with lymphadenectomy is recommended following endoscopic submucosal dissection (ESD) due to the risk of lymph-node metastasis for resected cancers. However, when lymphatic flows remain unchanged after ESD, a minimally invasive function-preserving surgery based on the sentinel node (SN) concept may be applicable. In this study, using porcine survival models, we aimed to investigate whether gastric lymphatic flows were modified following ESD.

Methods Twelve pigs, each with one simulating lesion $3 \mathrm{~cm}$ in size, were used. Indocyanine green (ICG) fluid was endoscopically injected into the submucosa in four quadrants surrounding the lesion. Following laparoscopic observation of lymphatic flows, the lesions were resected by ESD. After 4 weeks, ICG fluid was injected in four quadrants surrounding the scar and lymphatic flows were observed in the same manner as the initial procedure. The distribution of lymphatic flows, including stained SNs, was compared.

Results In ten lesions (83.3\%), the distribution of flows remained unchanged. However, in one lesion, the flow along the right gastric epiploic artery (R-GEA) disappeared on the lesser curvature of the middle stomach. In addition, in one lesion, the flow along R-GEA emerged on the lesser curvature of the lower stomach.

Conclusions Our study revealed that, despite ESD, lymphatic flows remained unchanged in most parts of the stomach. The SN concept may be applied after ESD, except for lesions on the lesser curvature. However, in the case of the lesser curvature, special care must be given to the SN concept.
\end{abstract}

Keywords Sentinel lymph node $\cdot$ Early gastric cancer $\cdot$ Endoscopic submucosal dissection

Osamu Goto

o-goto@nms.ac.jp

Kyoko Nohara

knohara@hosp.ncgm.go.jp

1 Department of Surgery, Keio University School of Medicine, Tokyo, Japan

2 Division of Research and Development for Minimally Invasive Treatment, Cancer Center, Keio University School of Medicine, Tokyo, Japan

3 Department of Gastroenterology, Nippon Medical School, 1-1-5, Sendagi, Bunkyo-ku, Tokyo 113-8603, Japan

4 Second Department of Surgery, Hamamatsu University School of Medicine, Shizuoka, Japan

5 Present Address: Department of Surgery, National Center for Global Health and Medicine, 1-21-1, Toyama, Shinjuku-ku, Tokyo 162-8655, Japan

\section{Introduction}

Endoscopic submucosal dissection (ESD) has been widely used for the curative treatment of limited early gastric cancer (EGC). The fifth Guideline, edited by the Japanese Cancer Association, proposes endoscopic resection (ER), including ESD, as a standard treatment for differentiated adenocarcinoma. Specifically, the latter is characterized by a tumor depth within the mucosa (T1a) without ulcerative findings, while the diameter is of $3 \mathrm{~cm}$ or less with ulcerative findings. In addition, the guidelines propose that a $2 \mathrm{~cm}$ or less $\mathrm{T} 1 \mathrm{a}$ undifferentiated adenocarcinoma without ulcerative findings can be cured by ESD as an expanded indication [1].

On the other hand, in case of resected cancers with possible lymph-node metastasis or in the presence of an advanced cancer, standard gastrectomy with lymphadenectomy is recommended following ESD. However, EGC demonstrates 
limited lymph-node metastasis and an excellent survival rate. This is due to the fact that, in most cases, an additional surgery reveals the absence of lymph-node metastasis and a local residual tumor $[2,3]$. Such an approach may seem to be an excessive treatment for patients with pathologically node-negative EGC. It has been shown that gastrectomy with lymph-node dissection decreases patients' quality of life (QOL) [4, 5]. Therefore, sentinel lymph-node navigation surgery (SNNS) is an appealing therapeutic option and has become the new paradigm in EGC treatment [6-9].

A sentinel node ( $\mathrm{SN}$ ) is defined as the first drainage node from the primary tumor. If SNs are metastases' free, theoretically, further lymph nodes should also have no metastases. This concept has been already applied for breast cancer and malignant melanoma [10, 11]. However, for EGC, it has been controversial for years. Based on the SN concept, a recently reported meta-analysis and prospective multicenter trial of SNNS for EGC have shown acceptable SN detection rates and accuracy of metastases diagnosis [8, 12]. However, to date, it has not been clarified whether the SNNS concept is applicable to EGC even after ESD. This is due to the fact that it remains unknown if the condition of lymphatic flow and the distribution of SNs remain unchanged even after ESD. Currently, a retrospective report has shown that the SN concept could be applied to tumors following ER [13]. However, a direct comparison of lymphatic conditions preand post-ER status is not available.

To overcome this limitation, in the present study, we hypothesized that $\mathrm{SN}$ basins would not be influenced by ESD. We investigated whether lymphatic flows, including SNs, would change following ESD using in vivo survival models to clarify if the SN concept could be applicable to cases following ER and to validate the finding for future clinical introduction.

\section{Materials and methods}

\section{Preparation}

In terms of both potential scientific contribution and ethics in animal protection, the study was approved by the institutional review board of the Laboratory Animal Center at Keio University School of Medicine (IRB no.14067). A survival experiment was performed in 12 female pigs weighing approximately $15-25 \mathrm{~kg}$ each in an animal research facility at Keio University School of Medicine. Surgery was performed under general anesthesia. The latter was induced by intramuscular administration of midazolam $(2 \mathrm{mg} / \mathrm{kg})$ and medetomidine $(0.1 \mathrm{mg} / \mathrm{kg})$, and maintained by sebofururen inhalation. Intramuscular injection of cefazolin $(0.5 \mathrm{~g})$ sodium hydrate was administered just before commencing the procedure.

\section{Setup for laparoscopic observation}

The surgeon stood on the left side of the porcine, whereas the first assistant and the laparoscopist stood on the right side of the porcine. A camera port was inserted by open method into the superior umbilical site. Subsequently, after inserting a laparoscope under a pneumoperitoneum of $10 \mathrm{mmHg}$, three 5-mm ports were placed in the right upper, right lower, and left upper quadrants, respectively.

\section{Procedure of first laparoscopic observation}

Each pig had one simulated lesion. These were created on the: anterior wall; posterior wall; greater curvature; lesser curvature in the upper, middle, and lower thirds of the stomach. Following transoral insertion of a flexible endoscope through an overtube, mucosal markings $3 \mathrm{~cm}$ in diameter were placed around the simulated lesion, with the use of the tip of a Dual knife (Olympus, Co., Ltd., Tokyo, Japan). Subsequently, $0.5 \mathrm{ml}$ of $0.5 \%$ indocyanine green (ICG) solution was injected into the submucosal layer of the stomach wall in four quadrants surrounding the simulated lesion. Ten minutes post-injection, we laparoscopically observed the SN and lymphatic flows using a near-infrared fluorescent contrast imaging system (VISERA Pro®; Olympus, Co., Ltd., Tokyo, Japan), with recording system (Fig. 1a-d). The distribution of lymphatic flows, including SNs, was divided into five basins: right gastric artery (R-GA); left gastric artery (L-GA); right gastroepiploic artery (R-GEA); left gastroepiploic artery (L-GEA); posterior gastric artery (P-GA). Subdivision was performed according to the location of feeding arteries $[14,15]$.

\section{Procedure of ESD}

Following observation of SN and lymphatic flows, ESD was conducted for the simulated lesion. As described in the other reports, a standard ESD procedure using the Dual knife (Olympus, Co., Ltd., Tokyo, Japan) was performed [16]. Post-resection, all the port sites were closed and the pigs were allowed to recover from anesthesia. All pigs were allowed to eat from postoperative day 1 and were administered antibiotics (cefazorin $0.5 \mathrm{~g}$ ) perorally for 3 days after ESD.

\section{Procedure of second laparoscopic observation}

At 4 week post-ESD, SN and lymphatic flows were reobserved. After the setting of laparoscopy and endoscopy in the same manner as the first observation, we evaluated the ESD scar endoscopically. Subsequently, 

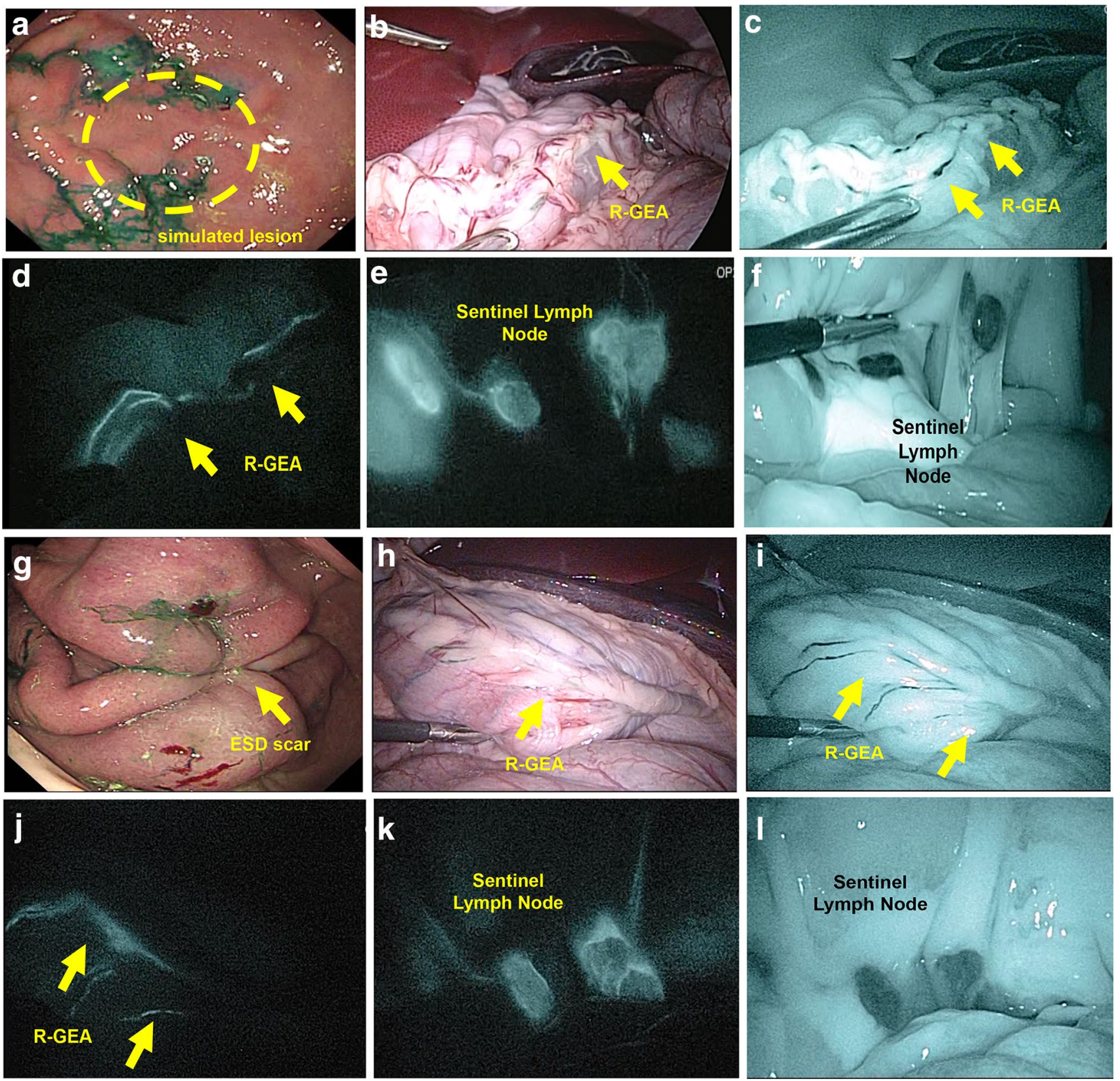

Fig. 1 Endoscopic and laparoscopic images in this experiment. a Before endoscopic submucosal dissection (ESD), indocyanine green (ICG) solution was injected into the submucosal layer of the stomach wall in four quadrants surrounding the lesion. b-f Following the injection, lymphatic flows and $\mathrm{SN}$ basins were laparoscopically observed using near-infrared fluorescent contrast imaging system
(VISERA Pro®; Olympus, Co., Ltd., Tokyo, Japan). g At 4 week post-ESD, ICG solution was injected into the normal submucosal layer in four quadrants surrounding the ESD scar. h-l Following the injection, lymphatic flows and SN basins were laparoscopically observed using the near-infrared fluorescent contrast imaging system

while recording videos (Fig. $1 \mathrm{e}-\mathrm{h}$ ). After the whole procedure, all pigs were sacrificed with intravenous injection of potassium chloride solution. By observing recording videos, we compared $\mathrm{SN}$ basins. the submucosal layer in four quadrants surrounding the scar was injected with $0.5 \mathrm{ml}$ of $0.5 \%$ ICG solution. Similarly, to the first experiment, $10 \mathrm{~min}$ post-injection, we observed the SN and lymphatic flows laparoscopically, 


\section{Results}

All pigs survived the procedures without adverse events $(12 / 12,100 \%)$. The average specimen size was $36 \mathrm{~mm}$ (range $28-50 \mathrm{~mm}$ ), and the average duration of ESD procedures was $24 \mathrm{~min}$ (range 15-39 min) (Table 1).

In 10 cases, SN basins' distribution was unchanged after ESD (83\%) (Table 2). On the contrary, in two cases on the lesser curvature, changes were observed. In the upper part of the stomach, SN basins were detected as follows: L-GEA and L-GA on the anterior wall; R-GEA, L-GEA, and L-GA on the greater curvature; P-GA on the posterior wall. In the middle part of the stomach, SN basins were detected as follows: R-GEA and L-GEA on the anterior wall; L-GA, L-GEA, and R-GEA on the greater curvature; L-GA and L-GEA on the posterior wall. In the lower part of the stomach, SN basins were detected as follows: R-GEA and L-GA on the anterior wall; R-GEA on the greater curvature; L-GA and R-GEA on the posterior wall. As to the lesser curvature in the middle third part, R-GEA, L-GA, and No7, 8a lymph node were detected as SN basin pre-ESD, but lymphatic flow along R-GEA was not observed in that area post-ESD (Fig. 2). Another case on the lesser curvature in the lower third part, R-GA, L-GA, and No7, 8a lymph node had been observed before ESD. In addition,
Table 1 Outcomes of endoscopic submucosal dissection in in vivo porcine models

\begin{tabular}{llllllll}
\hline No & Location & Circumference & $\begin{array}{l}\text { ESD dura- } \\
\text { tion (min) }\end{array}$ & $\begin{array}{l}\text { En bloc } \\
\text { resection }\end{array}$ & $\begin{array}{l}\text { Intraoperative } \\
\text { complication }\end{array}$ & $\begin{array}{l}\text { Longest } \\
\text { diameter(mm) }\end{array}$ & $\begin{array}{l}\text { Shortest } \\
\text { diameter(mm) }\end{array}$ \\
\hline 1 & U & Ant & 15 & Yes & None & 30 & 28 \\
2 & U & Gre & 30 & Yes & None & 47 & 37 \\
3 & U & Post & 13 & Yes & None & 30 & 20 \\
4 & U & Less & 39 & Yes & None & 27 & 20 \\
5 & M & Ant & 18 & Yes & None & 35 & 35 \\
6 & M & Gre & 23 & Yes & None & 50 & 48 \\
7 & M & Post & 14 & Yes & None & 44 & 28 \\
8 & M & Less & 34 & Yes & None & 37 & 34 \\
9 & L & Ant & 15 & Yes & None & 35 & 30 \\
10 & L & Gre & 39 & Yes & None & 34 & 23 \\
11 & L & Post & 31 & Yes & None & 38 & 24 \\
12 & L & Less & 22 & Yes & None & 28 & 26 \\
\hline
\end{tabular}

$U$ upper, $M$ middle, $L$ lower third of the stomach, Ant anterior wall, Gre greater curvature, Post posterior wall, Less lesser curvature of the stomach

Table 2 Lymph-node basin following ESD for simulated lesion

\begin{tabular}{|c|c|c|c|c|c|}
\hline \multirow[t]{2}{*}{ No. } & \multicolumn{2}{|l|}{ Before ESD } & \multicolumn{2}{|l|}{ After ESD } & \multirow[t]{2}{*}{ Changes after ESD } \\
\hline & Identified nodes & Node basin & Identified nodes & Node basin & \\
\hline 1 & None & L-GA,L-GEA & None & L-GA, L-GEA & No \\
\hline 2 & $6,7,8 \mathrm{a}$ & L-GA,L-GEA, R-GEA & $6,7,8 \mathrm{a}$ & $\begin{array}{l}\text { L-GA, L-GEA, } \\
\text { R-GEA }\end{array}$ & No \\
\hline 3 & $11 \mathrm{p}$ & Post-GA & $11 \mathrm{p}$ & Post-GA & No \\
\hline 4 & $7,8 \mathrm{a}$ & L-GA,R-GA & $7,8 \mathrm{a}$ & L-GA, R-GA & No \\
\hline 5 & 6,7 & L-GA,R-GEA & 6,7 & L-GA, R-GEA & No \\
\hline 6 & $7,8 \mathrm{a}$ & L-GA, L-GEA, R-GEA & $7,8 \mathrm{a}$ & $\begin{array}{l}\text { L-GA, L-GEA, } \\
\text { R-GEA }\end{array}$ & No \\
\hline 7 & 7 & L-GA, L-GEA & 7 & L-GA, L-GEA & No \\
\hline 8 & $7,8 \mathrm{a}$ & L-GA, R-GA, R-GEA & $7,8 \mathrm{a}$ & L-GA, R-GA & Loss of R-GEA \\
\hline 9 & $6,7,8 \mathrm{a}$ & L-GA, R-GEA & $6,7,8 \mathrm{a}$ & L-GA, R-GEA & No \\
\hline 10 & $6,8 \mathrm{a}$ & R-GEA & $6,8 \mathrm{a}$ & R-GEA & No \\
\hline 11 & $6,7,8 \mathrm{a}$ & L-GA, R-GEA & $6,7,8 \mathrm{a}$ & L-GA, R-GEA & No \\
\hline 12 & $7,8 \mathrm{a}$ & L-GA, R-GA & $6,7,8 \mathrm{a}$ & $\begin{array}{l}\text { L-GA, R-GA, } \\
\text { R-GEA }\end{array}$ & $\begin{array}{l}\text { R-GEA, No6 } \\
\text { emerged }\end{array}$ \\
\hline
\end{tabular}

$U$ upper, $M$ middle, $L$ lower third of the stomach, Ant anterior wall, Gre greater curvature, Post posterior wall, Less lesser curvature of the stomach, $L$-GEA left gastroepiploic artery, $L-G A$ left gastric artery, $R$ - $G E A$ right gastroepiploic artery, $R$ - $G A$ right gastric artery 
although R-GEA and No6 lymph node had not been detected before ESD, they emerged as SN basin after ESD (Fig. 3).

\section{Discussion}

In the present study, we confirmed that almost all lymphnode basins around the stomach remained unchanged following ESD in an in vivo survival model. We observed
post-ESD changes in only two cases in lesser curvature. To the best of our knowledge, this is the first report evaluating the possible changes of lymphatic flows following ESD using in vivo survival models.

In a retrospective report by Mayanagi et al. by comparing additional surgical cases after ER with ER-negative surgical cases, it was shown that $\mathrm{SN}$ basin was not greatly affected by ER from the results of SN basin [13]. However, they reported that the SN concept had to be carefully evaluated
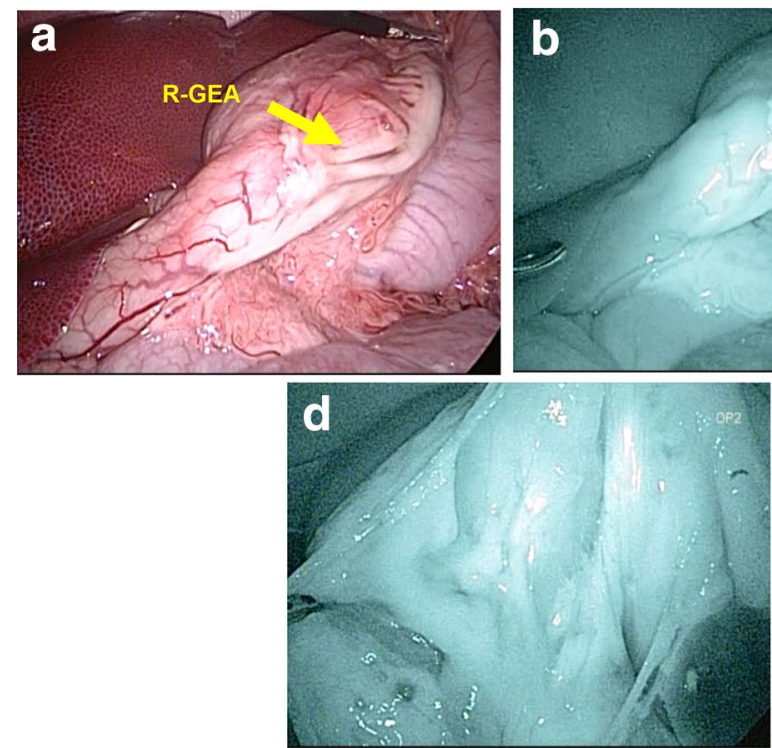

Fig. 2 The case on the lesser curvature in the middle part of the stomach. a-c Before endoscopic submucosal dissection (ESD), R-GEA was detected when ICG solution was injected into the submucosal
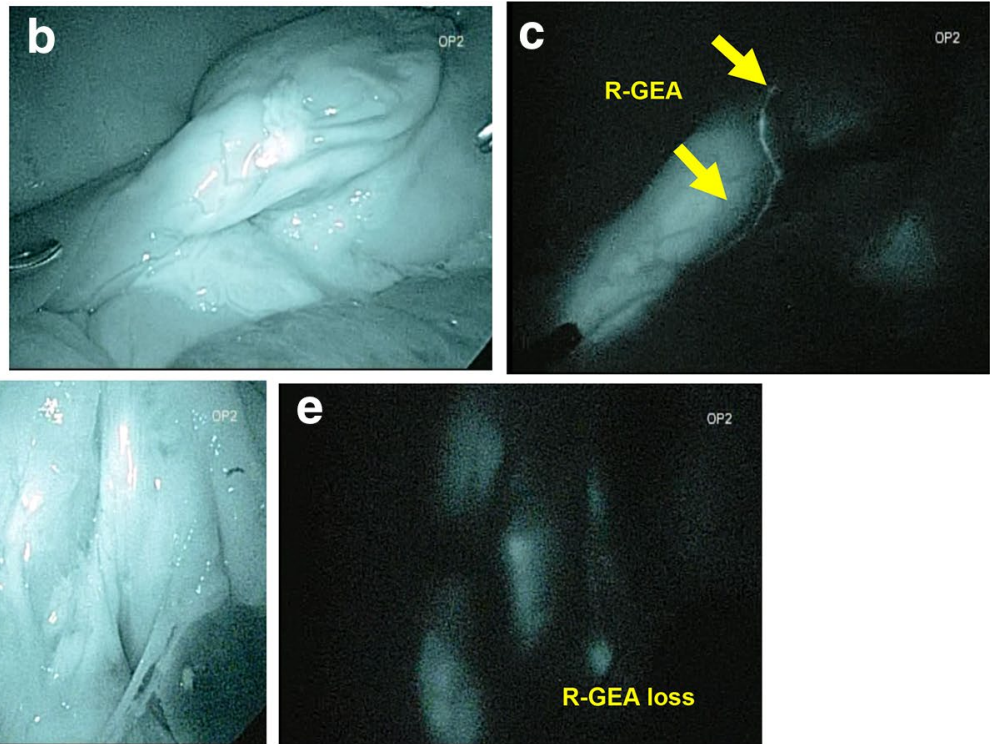

layer of the stomach wall in four quadrants surrounding the lesion on the lesser curvature in the middle part. d, e Lymphatic flow along R-GEA was not identified after ESD
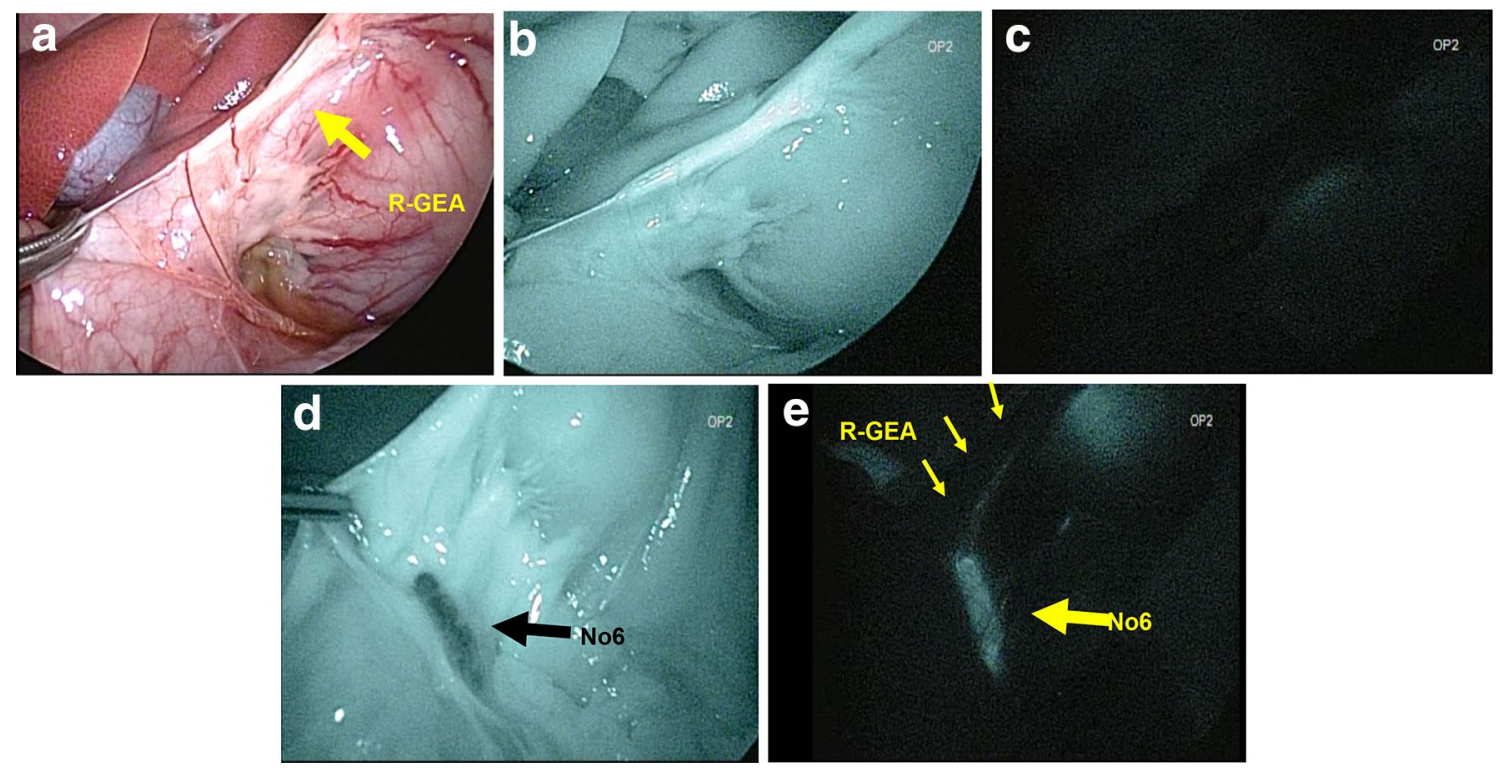

Fig. 3 The case on the lesser curvature in the lower part of the stomach. a-c Before endoscopic submucosal dissection (ESD), R-GEA and No6 lymph node were not detected after ICG injection. d, e R-GEA and No6 lymph node emerged after ESD 
because of the varied SN distribution when the tumor was located in the stomach's middle or lower third. However, to date, it still remains unknown whether ER modifies lymphatic flows. In the present study, we covered a whole area of the stomach by creating 12 lesions. These were located on the anterior wall; posterior wall; greater curvature; lesser curvature in the upper, middle, and lower thirds of the stomach. Since the distribution of SNs' basins is known to be relatively limited to the periphery of the stomach, in this study, we observed them on each different lesion. In all cases, lymph-node basins were not greatly affected by the previous ESD, except for two cases located in the lesser curvature.

In the present study, SN basin changed after ESD on the lesser curvature in the middle and lower parts of the stomach. When the tumor was located in the stomach's lesser curvature, SN distribution was changeable according to preceding ER. A retrospective study of SN mapping by Kinami et al. showed that the gastric lymphatic basins were divided into five directions following the course of the main arteries as follows: left gastric artery area, right gastric artery area, left gastroepiploic artery area, right gastroepiploic artery area, and posterior gastric artery area. The authors reported that L-GA was the most important lymphatic compartment regardless of the location of cancer on the long axis [15]. In this report, it was shown that the afferent lymphatics of cancer located in the middle part of the stomach had flowed in various directions. Accordingly, using the ready-made lymphatic networks, new connections between the primary site and other $\mathrm{SN}$ basins could be easily created in the L-GA dominant area. Tokunaga et al. also reported that the major lymphatic drainage routes of the stomach were associated with L-GA and R-GEA [17]. Furthermore, according to another previous study on 489 patients, SN distribution in EGCs varied on the lesser curvature in the middle and lower parts of the stomach, while it gathered along the L-GA almost all of EGCs on the lesser curvature in the upper part of the stomach [18]. In the present study, $16 \%$ and $12 \%$ of the lymphatic basins in the middle part and $42 \%$ and $44 \%$ of those in the lower part on the lesser curvature flowed along the R-GEA and R-GA, whereas the other lymphatic basins flowed along the L-GA. Thus, SN distribution varied in the middle and lower parts on the lesser curvature. Aoyama et al. reported that patients with tumors in regions covered by both L-GA and R-GEA, such as the those located at the lesser curvature in the lower body, could not be considered good candidates for local resection with the SN concept [19]. Furthermore, an additional explanation of our results might be linked to the omentum anatomy. Specifically, the lesser omentum widely connects to the stomach wall from the anterior side to the posterior side [20-22]. Because almost all of lymphatic flows go through the lesser omentum directly at the lesser curvature, the lymphatic basin might be easily influenced by ESD. However, even on the lesser curvature, no lymphatic changes were observed following ESD in the upper part of the stomach. In this case, both L-GA and R-GA were detected as SN basins. This finding coincided with the case in the lesser curvature on the lower part. Such finding might suggest that, in the porcine model, the length of the lesser curvature is too short to completely simulate the human stomach. Specifically, in the human stomach, it would be hard to expect lymphatic flows from the upper toward the lower part (R-GA) [11]. Therefore, it is not possible to easily conclude that $\mathrm{SN}$ basin is unchanged after ER in the upper area on the lesser curvature, even in human cases.

This is the first report examining the lymphatic changes following ESD with the use of in vivo survival models. If the SN concept was acceptable for cases post-ER, several function-preserving surgeries, such as partial resection, segmental gastrectomy, pylorus-preserving gastrectomy, and proximal gastrectomy for EGC, would be appropriate for a higher number of cases involving minimal lymph-node dissection [12, 23, 24]. Of note, however, in our study, the feasibility of the SN concept after ESD cases is not clear in the case of the lesser curvature. In Japan, the number of ESD is expected to increase, and the SNNS concept following ESD would be acceptable if results are obtained from a multicenter prospective study.

The following section reports some limitations of our study. First, the number of porcine models was small. We performed one ESD on each swine to avoid influencing lymphatic changes by another ESD. Second, it is unclear whether lymphatic flows changed, particularly in cases with ulceration. Therefore, ulcers' appearance should be evaluated to determine an adequate treatment strategy including SNNS for EGC. Third, whether lymphatic flows around a real tumor changed and how a post-ESD ulcer, including its surrounding lymphatic ducts, healed remain unknown [25]. Further studies are necessary to verify the pathological changes, including lymphatic flow around the tumor and the ESD scar. Histologic samples were needed to investigate the pathological changes both before and after ESD. Performing sample collection before ESD could influence the lymphatic flow around the ESD scar. Obtaining histologic samples from the same part before and after ESD was challenging; this was a limitation of our study. Fourth, whether to apply our results to the human stomach remains undetermined, because the porcine stomach wall is much thicker than that of the human stomach. Horii $\mathrm{J}$ et al. reported that the mucosal layers in the porcine stomach were considerably thicker than those in the human stomach in the lower areas, particularly in the greater curvature in the lower part [26]. In the present study, the thicknesses of the submucosal and muscle layers did not seem different. Thus, the influence of cauterization 
on the lymphatic path outside and in the porcine stomach was not thought to be much different from that outside and in the human stomach.

In conclusion, in almost all cases, $\mathrm{SN}$ basins were not influenced by ESD in this porcine survival study. However, it appeared that the lymphatic change potential post-ER differ when the tumor is localized on the lesser curvature. Our results suggest that caution is needed for the indication of the SN concept when taking into consideration the lesser curvature. We may be able to apply SNNS even to EGC following non-curative ESD, with the exception of cases located on the lesser curvature.

Funding This study was partly supported by a Grant-in-Aid for Scientific Research (C) from the Ministry of Education, Culture, Sports, Science and Technology in Japan in 2015-2017 (Grant No. 15K09061).

\section{Compliance with ethical standards}

Conflict of interest The authors declare that they have no conflicts of interest.

Ethical standards All institutional and national guidelines for the care and use of laboratory animals were followed.

Human/animal rights The study was approved by the institutional review board of the Laboratory Animal Center at Keio University School of Medicine (IRB no.14067). This article does not contain any studies with human performed by any of the authors.

\section{References}

1. Japanese gastric cancer treatment guidelines. Gastric Cancer. 2014; ver. 4:2017;20(1):1-19.

2. An JY, Baik YH, Choi MG, Noh JH, Sohn TS, Kim S. Predictive factors for lymph node metastasis in early gastric cancer with submucosal invasion: analysis of a single institutional experience. Ann Surg. 2007;246(5):749-53.

3. Sunagawa H, Kinoshita T, Kaito A, Shibasaki H, Kaneko K, Ochiai A, et al. Additional surgery for non-curative resection after endoscopic submucosal dissection for gastric cancer: a retrospective analysis of 200 cases. Surg Today. 2017;47(2):202-9.

4. Davies J, Johnston D, Sue-Ling H, Young S, May J, Griffith J, et al. Total or subtotal gastrectomy for gastric carcinoma? A study of quality of life. World J Surg. 1998;22(10):1048-55.

5. Yamashita K, Kurokawa Y, Yamamoto K, Hirota M, Kawabata R, Mikami J, et al. Risk factors for poor compliance with adjuvant S-1 chemotherapy for gastric cancer: a multicenter retrospective study. Ann Surg Oncol. 2017;24(9):2639-45.

6. An JY, Min JS, Lee YJ, Jeong SH, Hur H, Han SU, et al. Which factors are important for successful sentinel node navigation surgery in gastric cancer patients? Analysis from the SENORITA prospective multicenter feasibility quality control trial. Gastroenterol Res Pract. 2017;2017:1732571.

7. Kitagawa Y, Fujii H, Mukai M, Kubota T, Ando N, Watanabe $\mathrm{M}$, et al. The role of the sentinel lymph node in gastrointestinal cancer. Surg Clin N Am. 2000;80(6):1799-809.
8. Kitagawa Y, Takeuchi H, Takagi Y, Natsugoe S, Terashima M, Murakami N, et al. Sentinel node mapping for gastric cancer: a prospective multicenter trial in Japan. J Clin Oncol. 2013;31(29):3704-10.

9. Takahashi N, Nimura H, Fujita T, Mitsumori N, Shiraishi N, Kitano S, et al. Laparoscopic sentinel node navigation surgery for early gastric cancer: a prospective multicenter trial. Langenbecks Arch Surg. 2017;402(1):27-32.

10. Morton DL, Wen DR, Wong JH, Economou JS, Cagle LA, Storm FK, et al. Technical details of intraoperative lymphatic mapping for early stage melanoma. Arch Surg. 1992;127(4):392-9.

11. Veronesi U, Paganelli G, Viale G, Luini A, Zurrida S, Galimberti $\mathrm{V}$, et al. A randomized comparison of sentinel-node biopsy with routine axillary dissection in breast cancer. $\mathrm{N}$ Engl $\mathrm{J}$ Med. 2003;349(6):546-53.

12. Takeuchi H, Kitagawa Y. Minimally invasive function-preserving surgery based on sentinel node concept in early gastric cancer. Transl Gastroenterol Hepatol. 2016;1:23.

13. Mayanagi S, Takeuchi H, Kamiya S, Niihara M, Nakamura R, Takahashi T, et al. Suitability of sentinel node mapping as an index of metastasis in early gastric cancer following endoscopic resection. Ann Surg Oncol. 2014;21(9):2987-93.

14. Miwa K, Kinami S, Taniguchi K, Fushida S, Fujimura T, Nonomura A. Mapping sentinel nodes in patients with early-stage gastric carcinoma. Br J Surg. 2003;90(2):178-82.

15. Kinami S, Fujimura T, Ojima E, Fushida S, Ojima T, Funaki H, et al. PTD classification: proposal for a new classification of gastric cancer location based on physiological lymphatic flow. Int $\mathbf{J}$ Clin Oncol. 2008;13(4):320-9.

16. Yahagi N, Uraoka T, Ida Y, Hosoe N, Nakamura R, Kitagawa Y, Ogata H, Hibi T. Endoscopic submucosal dissestion using the flex and the dual knives. Tech Gastrointest Endosc. 2011; 13:74-8.

17. Tokunaga M, Ohyama S, Hiki N, Fukunaga T, Yamada K, Sano $\mathrm{T}$, et al. Investigation of the lymphatic stream of the stomach in gastric cancer with solitary lymph node metastasis. World J Surg. 2009;33(6):1235-9.

18. Takeuchi H, Goto O, Yahagi N, Kitagawa Y. Function-preserving gastrectomy based on the sentinel node concept in early gastric cancer. Gastric Cancer. 2017;20(Suppl 1):53-9.

19. Aoyama J, Kawakubo H, Goto O, Nakahara T, Mayanagi S, Fukuda K, et al. Potential for local resection with sentinel node basin dissection for early gastric cancer based on the distribution of primary sites. Gastric Cancer. 2018;1:1-6.

20. Hwang SE, Cho BH, Hirai I, Kim HT, Kim JH, Fujimiya M, et al. Topographical anatomy of Spiegel's lobe and its adjacent organs in mid-term fetuses: its implication on the development of the lesser sac and adult morphology of the upper abdomen. Clin Anat. 2010;23(6): 712-9.

21. Doom M, de Rooster H, van Bergen T, Gielen I, Kromhout K, Simoens P, et al. Morphology of the canine omentum part 1: arterial landmarks that define the omentum. Anat Histol Embryol. 2016;45(1):37-43.

22. Zhao Z, Liu S, Li Z, Hou J, Wang Z, Ma X, et al. Sectional anatomy of the peritoneal reflections of the upper abdomen in the coronal plane. J Comput Assist Tomogr. 2005;29(4):430-7.

23. Nunobe S, Sasako M, Saka M, Fukagawa T, Katai H, Sano T. Symptom evaluation of long-term postoperative outcomes after pylorus-preserving gastrectomy for early gastric cancer. Gastric Cancer. 2007;10(3):167-72.

24. Tokunaga M, Hiki N, Ohyama S, Nunobe S, Miki A, Fukunaga $\mathrm{T}$, et al. Effects of reconstruction methods on a patient's quality of life after a proximal gastrectomy: subjective symptoms evaluation using questionnaire survey. Langenbecks Arch Surg. 2009;394(4):637-41. 
25. Arantes V, Uedo N, Pedrosa MS, Tomita Y. Clinical relevance of aberrant polypoid nodule scar after endoscopic submucosal dissection. World J Gastrointest Endosc. 2016;8(17):628-34.

26. Horii J, Goto O, Shimoda M, Sasaki M, Fujimoto A, Ochiai Y, et al. Which part of a porcine stomach is suitable as an animal training model for gastric endoscopic submucosal dissection? Endoscopy. 2016;48(2):188-93.

Publisher's Note Springer Nature remains neutral with regard to jurisdictional claims in published maps and institutional affiliations. 